\title{
Comportamento higroscópico da polpa de graviola em pó obtida por secagem em spray dryer
}

\author{
Hygroscopic behaviour of spray dried soursop pulp powder
}

\author{
Carlos Eliardo Barros Cavalcante ${ }^{1 *}$ (1), Sueli Rodrigues², Marcos Rodrigues Amorim Afonso², José Maria Correia Costa \\ 1/nstituto Federal de Educação, Ciência e Tecnologia do Ceará (IFCE), Sobral/CE - Brasil \\ 2Universidade Federal do Ceará (UFC), Centro de Ciências Agrárias, Departamento de Tecnologia de Alimentos, Fortaleza/CE - Brasil
}

\section{${ }^{*}$ Corresponding Author}

Carlos Eliardo Barros Cavalcante, Instituto Federal de Educação, Ciência e Tecnologia do Ceará (IFCE), Campus Sobral, Avenida Doutor Guarany, 317, Derby Clube, CEP: 62040-730, Sobral/CE - Brasil, e-mail: eliardo@ifce.edu.br

Cite as: Hygroscopic behaviour of spray dried soursop pulp powder. Braz. J. Food Technol., v. 21, e2017121, 2018.

Received: Aug. 04, 2017; Accepted: Feb. 02, 2018

\section{Resumo}

Produtos em pó são caracterizados por sua praticidade e longa vida útil, no entanto as polpas de fruta em pó apresentam alta higroscopicidade e tendência à aglomeração devido à sua natureza hidrofílica. Sua estabilidade pode ser estudada por meio das isotermas de equilíbrio higroscópico. A polpa de graviola, por sua vez, apresenta grande potencial econômico e industrial e destaca-se em relação às outras polpas devido a seus excelentes atributos sensoriais. O presente trabalho teve como objetivo a caracterização físico-química e higroscópica da polpa de graviola em pó e avaliou seu comportamento higroscópico através da análise de isotermas de adsorção, obtidas por ajuste dos dados experimentais através dos modelos matemáticos de GAB, BET, Henderson e Oswin nas temperaturas de $25^{\circ} \mathrm{C}, 30^{\circ} \mathrm{C}, 35^{\circ} \mathrm{C}$ e $40^{\circ} \mathrm{C}$. Os resultados demonstraram que a polpa de graviola em pó é uma boa fonte de ácido ascórbico, ligeiramente higroscópica e abundante em formação de caking. O modelo GAB foi o que melhor representou as isotermas de adsorção, com coeficientes de determinação $\left(R^{2}\right)$ maiores que 0,99 e erros médios (E) menores que $5 \%$, apresentando comportamento do tipo III, característico dos alimentos ricos em açúcar e mostrando que é necessário ter cuidado no armazenamento desse produto em ambientes com umidade relativa acima de $60 \%$.

Palavras-chave: Atomização; Fruta em pó; Qualidade do pó; Atividade de água; Higroscopicidade; Modelagem matemática.

\section{Abstract}

Powdered products are characterized by their convenience and long shelf life; however, powdered fruit pulps are highly hygroscopic and tend to agglomerate due to their hydrophilic nature. The stability of fruit pulp powders can be studied by means of hygroscopic equilibrium isotherms. On the other hand, soursop pulp presents great economic and industrial potential and stands out from other pulps due to its excellent sensory attributes. This study aimed to evaluate the physicochemical and hygroscopic characteristics of the powdered soursop pulp processed by spray drying, and evaluate its hygroscopic behaviour by analysing the adsorption isotherms by fitting the experimental data to the GAB, BET, Henderson and Oswin mathematical models at $25^{\circ} \mathrm{C}, 30^{\circ} \mathrm{C}, 35^{\circ} \mathrm{C}$ and $40^{\circ} \mathrm{C}$. The results demonstrated that the soursop pulp powder was a good source of ascorbic acid, slightly hygroscopic and showed a lot of caking. The GAB model presented the best fit to the adsorption isotherms, with a coefficient of determination $\left(\mathrm{R}^{2}\right)$ above 0.99 and mean errors (E) below $5 \%$, presenting Type III behaviour, characteristic of sugar rich foods, and revealing that care should be taken when storing this product in environments with a relative humidity above $60 \%$.

Keywords: Spray-drying; Fruit powder; Powder quality; Water activity; Hygroscopicity; Mathematical modelling. 


\section{Introdução}

A graviola in natura apresenta baixa expressão comercial diante de outras frutas. No entanto, a transformação da fruta em polpa faz com que a de sabor graviola seja a quinta mais vendida na região Nordeste do Brasil, com total de $12 \%$, ficando em volume atrás apenas das polpas de acerola, goiaba, maracujá e caju (LEMOS, 2014). A alta demanda e interesse crescente, por parte do consumidor e das indústrias que utilizam o fruto como matéria-prima para produção de diferentes produtos, tornam a polpa de graviola promissora para ser aproveitada através de novas formas de consumo e comercialização.

Os secadores de aspersão, também conhecidos como spray dryers, são utilizados para secagem de soluções, suspensões, emulsões e pastas. Quando bem conduzida, a desidratação em spray dryer gera um produto altamente nutritivo, estável e versátil (PEDRO et al., 2010; FERRARI et al., 2012).

No caso da desidratação de polpas de frutas em spray dryer, o alto conteúdo de açúcares pode acarretar na obtenção de produtos com alta higroscopicidade e elevada pegajosidade, diminuindo assim o rendimento do processo (JAYA; DAS, 2004; FERRARI et al., 2012; OLIVEIRA et al., 2013b). Portanto, é fundamental a utilização de agentes carreadores com alto peso molecular antes da secagem em spray dryer, visando facilitar este processo e as operações de transporte e armazenamento (FERRARI et al., 2012). A maltodextrina é um dos principais aditivos utilizados para auxiliar a secagem de polpa de frutas em spray dryer, devido ao seu baixo custo e sua baixa higroscopicidade, evitando a aglomeração de partículas (FERRARI et al., 2012; CEBALLOS et al., 2012).

A utilização de agentes carreadores, como a maltodextrina, pode promover um melhor manuseio do produto final obtido, conferindo-Ihe maior proteção contra a adsorção de umidade do ambiente e tornando-o menos higroscópico (TONON et al., 2009). A higroscopicidade de um alimento está ligada à sua estabilidade física, química e microbiológica por isso, torna-se imprescindível o conhecimento do comportamento higroscópico desses produtos (OLIVEIRA et al., 2014).

Uma forma de se conhecer o comportamento higroscópico dos alimentos é através de suas isotermas de sorção. O conhecimento das isotermas de sorção de umidade dos alimentos auxilia na determinação do tempo de secagem, caracterização do produto, além da determinação de sua vida útil e do tipo de embalagem adequada para seu armazenamento (ALEXANDRE et al., 2007).

Diante destes fatores, o objetivo deste trabalho foi avaliar o comportamento higroscópico da polpa de graviola em pó, obtida por secagem em spray dryer, através da determinação de suas isotermas de adsorção e de sua caracterização físico-química.

\section{Material e métodos}

As polpas de graviola, não pasteurizadas e sem adição de conservantes, selecionadas para a realização deste estudo foram adquiridas em uma indústria de processamento de polpas de frutas localizada no município de Fortaleza - CE e encaminhadas para o Laboratório de Controle de Qualidade de Alimentos e Secagem do Departamento de Tecnologia de Alimentos da Universidade Federal do Ceará, local onde foram armazenadas nas condições de $-18{ }^{\circ} \mathrm{C}$ de temperatura e utilizadas de acordo com a necessidade experimental.

$O$ descongelamento foi realizado com as polpas ainda embaladas $6{ }^{\circ} \mathrm{C} \pm 2^{\circ} \mathrm{C}$ de temperatura, em refrigerador, por um período mínimo de 18 horas. Utilizou-se maltodextrina com dextrose equivalente (DE) 20 como coadjuvante de secagem na formulação da polpa de graviola para secagem na concentração de $17 \%(\mathrm{~m} / \mathrm{m})$.

Para a secagem em spray dryer, foram utilizadas as condições determinadas por Cavalcante et al. (2017); cuja avaliação da influência da temperatura do ar de secagem e da concentração de maltodextrina sobre as características da polpa determinou que a secagem a $169^{\circ} \mathrm{C}$ e $17 \%(\mathrm{~m} / \mathrm{m})$ de maltodextrina como adjuvante resulta em uma polpa com boas características físico-químicas e higroscópicas.

A secagem da polpa formulada ( $83 \%$ polpa e $17 \%$ maltodextrina) foi realizada em equipamento spray dryer modelo LM SMD 1.0 da marca Labmaq do Brasil, Ribeirão Preto, com a utilização de um bico aspersor de $1,2 \mathrm{~mm}$ de diâmetro, vazão de ar comprimido de 3,0 L. $\mathrm{min}^{-1}$, pressão de aspersão de 100 psi, vazão de alimentação de 0,5 L h ${ }^{-1}$, vazão de ar de secagem de $3,5 \mathrm{~m}^{3} \mathrm{~min}^{-1}$ e temperatura de entrada do ar de $169^{\circ} \mathrm{C}$. Parâmetros que, de acordo com Cavalcante et al. (2017), propiciam materiais com características físico-químicas e higroscópicas consideradas boas para pós alimentícios.

O processo de secagem foi realizado em triplicata e em cada repetição desidratou-se aproximadamente $1.000 \mathrm{~g}$ de polpa formulada sob condições mais uniformes possíveis para que não houvesse diferença entre as condições de trabalho.

A caracterização físico-química da polpa integral e da polpa em pó foi realizada em triplicata através das seguintes análises: a atividade da água determinada a $25{ }^{\circ} \mathrm{C}$ em higrômetro digital (Aqualab ${ }^{\circledR} 3 \mathrm{TE}$, Decagon, Pullman); e a umidade definida por infravermelho em determinador de umidade digital (ID50, Marte, São Paulo).

$\mathrm{O} \mathrm{pH}$ foi determinado através de um medidor de pH (Q400A, Quimis, São Paulo), calibrado periodicamente com soluções tampão de pH 4,0 e 7,0), a acidez titulável por titulação com $\mathrm{NaOH} 0,1 \mathrm{~N}$ e os resultados expressos em percentagem de ácido cítrico segundo a metodologia do IAL (2008). O conteúdo de vitamina C foi determinado através do método titulométrico baseado 
na redução do indicador 2,6-diclorofenolindofenol pelo ácido ascórbico e os resultados expressos em mg de ácido ascórbico por $100 \mathrm{~g}$ de amostra (CUNNIFF, 1997). A análise de cor foi determinada utilizando-se colorímetro (Chroma-Meter CR-410, Konica Minolta, Japão) com determinação da escala $\operatorname{CIE~} L^{*} a^{*} b^{*}$, no qual $L^{*}$ define a luminosidade $\left(L^{*}=0\right.$ preto e $L^{*}=100$ branco) e $a^{*} e$ $b^{*}$ são responsáveis pela cromaticidade $\left(+a^{*}\right.$ vermelho e $-a^{*}$ verde, $+b^{*}$ amarelo e $-b^{*}$ azul). A partir destes parâmetros, foram avaliadas as coordenadas cilíndricas $\mathrm{C}^{*}$ e $\mathrm{H}^{*}$, sendo que $\mathrm{C}^{*}$ define o croma e $\mathrm{H}^{*} \mathrm{o}$ ângulo de tom, a partir das Equações 1 e 2 (FERRARI et al., 2012)

$$
\begin{aligned}
& C^{*}=\left(a^{* 2}+b^{* 2}\right)^{1 / 2} \\
& H^{*}=\arctan \left(\frac{a^{*}}{b^{*}}\right)
\end{aligned}
$$

A determinação da densidade aparente foi realizada segundo a metodologia proposta por Goula e Adamopoulos (2010) com resultados expressos em $\mathrm{g} \mathrm{mL}^{-1}$.

A caracterização higroscópica da polpa de graviola em pó obtida por secagem em spray dryer foi realizada por meio das determinações de higroscopicidade (GOULA; ADAMOPOULOS, 2010), expressa em percentual de umidade adsorvida, grau de caking (JAYA; DAS, 2004) e solubilidade (CANO-CHAUCA et al., 2005), ambos expressos em percentual. Toda a caracterização higroscópica foi realizada em triplicata.

Para as determinações das isotermas de adsorção foi utilizado o método gravimétrico estático. Amostras, em triplicata, de 0,2 g do pó foram pesadas em cadinhos de alumínio previamente tarados e colocadas em ambiente com umidades relativas condicionadas por meio de diferentes soluções salinas saturadas, tais como $\mathrm{CH}_{3} \operatorname{COOK}\left(\mathrm{a}_{\mathrm{w}}=0,21\right)$, $\mathrm{K}_{2} \mathrm{CO}_{3}\left(\mathrm{a}_{\mathrm{w}}=0,44\right), \mathrm{NaBr}\left(\mathrm{a}_{\mathrm{w}}=0,58\right), \mathrm{SnCl}_{2}\left(\mathrm{a}_{\mathrm{w}}=0,76\right)$, $\mathrm{KCl}\left(\mathrm{a}_{\mathrm{w}}=0,84\right)$ e $\mathrm{BaCl}_{2}\left(\mathrm{a}_{\mathrm{w}}=0,90\right)$, preparadas de acordo com Greenspan (1977)

As amostras foram pesadas a cada 24 horas por um período de aproximadamente 13 dias, até atingirem peso constante e variação de peso inferior a 0,1\% (0,001 $\mathrm{g} \mathrm{g}^{-1}$ sólido), determinando-se, desta forma, a massa de equilíbrio. Após a detecção do equilíbrio, a atividade de água $\left(\mathrm{a}_{\mathrm{w}}\right)$ de cada amostra foi medida nas temperaturas de $25^{\circ} \mathrm{C}, 30^{\circ} \mathrm{C}, 35^{\circ} \mathrm{C}$ e $40^{\circ} \mathrm{C}$, em higrômetro digital (Aqualab ${ }^{\circledR}$ 3TE, Decagon, Pullman). Posteriormente, as amostras foram levadas para secagem em estufa a vácuo (modelo, marca e cidade) com temperatura de $70{ }^{\circ} \mathrm{C}$ para determinação da massa seca.

A umidade de equilíbrio $\left(X_{\text {eq }}\right)$ foi calculada segundo a Equação 3.

$X_{\text {eq }}=\frac{m_{o}-m_{s}}{m_{s}}$
Tabela 1. Modelos matemáticos utilizados para o ajuste das isotermas de adsorção da polpa de graviola em pó obtida por secagem em spray dryer.

Modelos
GAB $\quad X_{\text {eq }}=\frac{\text { Equações }}{\left(1-K \cdot a_{w}\right) \cdot\left(1-K \cdot a_{w}+C K \cdot a_{w}\right)}$
BET $\quad X_{\text {eq }}=\frac{X_{m} \cdot C \cdot a_{w}}{\left(1-a_{w}\right)} \cdot\left[\frac{1-(n+1) \cdot\left(a_{w}\right)^{n}+n \cdot\left(a_{w}\right)^{n+1}}{1-(1-C) \cdot a_{w}-C \cdot\left(a_{w}\right)^{n+1}}\right]$
HENDERSON
$X_{\text {eq }}=\left[\frac{-\ln \left(1-a_{w}\right)}{b}\right]^{\frac{1}{a}}$
OSWIN

Xeq: umidade de equilíbrio ( $g$ de $\mathrm{H}_{2} \mathrm{O} \mathrm{g}^{-1}$ ); $\mathrm{X}$ : conteúdo de água na monocamada molecular ( $\mathrm{g}$ de $\mathrm{H}_{2}^{2} \mathrm{O} \mathrm{g}^{-1}$ ); $\mathrm{a}_{\mathrm{w}}^{\mathrm{m}}$ : atividade de água; C: constante relativa ao calor total de sorção da primeira camada; $\mathrm{K}$ : Constante de GAB relativa às multicamadas; $n$ : Constante de BET relativa às multicamadas; $a$ e $b$ : parâmetros de ajuste para os modelos de Henderson e Oswin.

uma vez que: $X_{\text {eq }}=$ umidade de equilíbrio, $\mathrm{g} \mathrm{g}^{-1} ; \mathrm{m}_{\mathrm{o}}=$ massa da amostra no equilíbrio, $\mathrm{g} ; \mathrm{m}_{\mathrm{s}}=$ massa da amostra seca, $\mathrm{g}$.

Para o ajuste dos dados experimentais na obtenção das isotermas de adsorção foram utilizados os modelos matemáticos apresentados na Tabela 1.

Os modelos foram ajustados utilizando o software Statistica 7.0 (STATSOFT, 2007) e seu nível de qualidade foi avaliado pelo coeficiente de correlação $\left(R^{2}\right)$ e pelo erro médio relativo (E) definido pela Equação 4.

$E=\frac{100}{n} \sum_{i-1}^{n} \frac{\left(M_{i}-M_{p}\right) \mid}{M_{i}}$

em que: $E$ = resíduo médio relativo (\%); $M_{i}=$ valor experimental; $M_{p}=$ valor predito pelo modelo; e $n$ = número de dados experimentais.

\section{Resultados e discussão}

$\mathrm{Na}$ Tabela 2 encontram-se os resultados das análises físico-químicas realizadas na polpa integral de graviola como para polpa em pó obtida por secagem em spray dryer a $169{ }^{\circ} \mathrm{C}$ de temperatura e $17 \%(\mathrm{~m} / \mathrm{m})$ de maltodextrina.

A polpa de graviola integral apresentou acidez em torno de 0,70\% de ácido cítrico, pH por volta de 3,50, teor de sólidos solúveis variando entre 9,6 ${ }^{\circ}$ Brix e teor de vitamina C de $37 \mathrm{mg} 100 \mathrm{~g}^{-1}$. Resultados similares foram relatados em outros trabalhos (LIMA et al., 2010; COSTA et al., 2014). Ainda com relação aos resultados encontrados, verificou-se que todos estavam de acordo 
Tabela 2. Caracterização físico-química da polpa de graviola integral (base úmida) e em pó (base seca) obtida por secagem em spray dryer, em $169^{\circ} \mathrm{C}$ de temperatura e $17 \%(\mathrm{~m} / \mathrm{m})$ de maltodextrina.

\begin{tabular}{|c|c|c|c|}
\hline \multicolumn{2}{|c|}{ Parâmetro analisado } & Polpa integral (b.u.) & Polpa em pó (b.s.) \\
\hline \multicolumn{2}{|c|}{ Acidez titulável (\% m/m de ácido cítrico) } & $0,70 \pm 0,02$ & $2,36 \pm 0,06$ \\
\hline \multicolumn{2}{|l|}{$\mathrm{pH}$} & $3,50 \pm 0,03$ & $3,69 \pm 0,08$ \\
\hline \multicolumn{2}{|c|}{ Sólidos solúveis totais ( ${ }^{\circ} \mathrm{Brix}$ ) } & $9,63 \pm 0,55$ & * \\
\hline \multicolumn{2}{|c|}{ Vitamina C (mg ácido ascórbico. $100 \mathrm{~g}^{-1}$ ) } & $37,79 \pm 0,09$ & $102,39 \pm 0,53$ \\
\hline \multirow{5}{*}{ Parâmetros de cor } & $L^{*}$ & $67,22 \pm 0,07$ & $68,03 \pm 0,02$ \\
\hline & $a^{*}$ & $-1,13 \pm 0,05$ & $-2,48 \pm 0,01$ \\
\hline & $b^{*}$ & $11,04 \pm 0,02$ & $6,40 \pm 0,02$ \\
\hline & $H_{0}^{*}$ & $95,55 \pm 0,02$ & $111,17 \pm 0,11$ \\
\hline & $C^{*}$ & $11,08 \pm 0,02$ & $6,87 \pm 0,02$ \\
\hline \multicolumn{2}{|c|}{ Umidade $(\% \mathrm{~m} / \mathrm{m})$} & $89,29 \pm 1,88$ & $1,39 \pm 0,06$ \\
\hline \multicolumn{2}{|c|}{ Atividade de água } & $0,98 \pm 0,01$ & $0,22 \pm 0,03$ \\
\hline \multicolumn{2}{|c|}{ Densidade aparente } & * & $0,53 \pm 0,02$ \\
\hline
\end{tabular}

*Análise não aplicada ao material devido seu estado físico.

com os teores preconizados pelos padrões de identidade e qualidade para este produto (BRASIL, 2000).

A polpa de graviola em pó apresentou acidez em torno de 2,36\% de ácido cítrico, pH por volta de 3,6, teor de vitamina C de 102 mg $100 \mathrm{~g}^{-1}$ e coloração branca com base nos baixos valores nos parâmetros $a^{*}, b^{*}, C^{*}$, elevado valor no parâmetro $\mathrm{H}_{0}{ }^{*}$ e considerável luminosidade. $\mathrm{O}$ alto valor de $L^{*}$ pode ser visto como um aspecto positivo das condições de secagem utilizadas, indicando que não houve escurecimento do pó.

Resultados semelhantes foram relatados por Costa et al. (2014) em estudo da secagem da polpa de graviola em spray dryer a uma temperatura de $120{ }^{\circ} \mathrm{C} \mathrm{e}$ concentrações de maltodextrina de 15\%, 30\% e 45\% (m/m) obtiveram pós claros com elevado parâmetro de $\mathrm{L}^{*} \mathrm{com}$ valores de $\mathrm{pH}$ e acidez titulável por volta de 3,7 e 3,1\% respectivamente.

A polpa de graviola em pó apresentou teor de umidade e atividade de água de 1,39\% e 0,22 respectivamente, permitindo-a ser classificada como estável em relação à deterioração bioquímica e microbiana. Sua densidade aparente foi de $0,53 \mathrm{~g} \mathrm{~mL}^{-1}$. A baixa densidade está associada ao baixo teor de umidade do material e ao uso de maltodextrina como adjuvante de secagem.

A formulação da polpa com maltodextrina elevou o teor de sólidos totais reduzindo o teor de umidade do material a ser desidratado, facilitando assim a obtenção de pós com menor teor de umidade e quanto menor for o teor de umidade menor será a densidade do material (CHEGINI; GHOBADIAN, 2005; QUEK et al., 2007).

$\mathrm{Na}$ Tabela 3 encontram-se os resultados médios para os parâmetros higroscópicos da polpa de graviola em pó.

A solubilidade foi o critério mais confiável para avaliar o comportamento do pó em solução aquosa
Tabela 3. Parâmetros higroscópicos da polpa de graviola em pó obtida por secagem em spray dryer, em $169^{\circ} \mathrm{C}$ de temperatura e $17 \%(\mathrm{~m} / \mathrm{m})$ de maltodextrina.

\begin{tabular}{cc} 
Parâmetro analisado & Polpa em pó \\
Solubilidade $(\%)$ & $62,46 \pm 0,40$ \\
Higroscopicidade $\left(\mathrm{g} .100 \mathrm{~g}^{-1}\right)$ & $10,01 \pm 0,45$ \\
Grau de Caking (\%) & $67,22 \pm 4,57$ \\
\hline
\end{tabular}

(CAPARINO et al., 2012) e a adição de maltodextrina como adjuvante de secagem na obtenção de polpas de fruta em pó resultou em pós com alta solubilidade. Diversos autores relataram uma solubilidade média maior que $80 \%$ em pós de polpa de fruta obtidos em spray dryer e adicionados de maltodextrina como adjuvante de secagem (CANO-CHAUCA et al., 2005; CAPARINO et al., 2012; CEBALLOS et al., 2012; FAZAELI et al., 2012; OLIVEIRA et al., 2013b). Diante isso, a solubilidade de $62 \%$ encontrada no presente trabalho foi considerada baixa.

A higroscopicidade e grau de caking da polpa de graviola em pó foram de 10,01 e 67,22\%, respectivamente e de acordo com a classificação proposta por GEA Niro Research Laboratory (2003) e pode ser classificada como ligeiramente higroscópica e de abundante formação de caking.

Costa et al. (2014) em estudo da secagem da polpa de graviola em spray dryer a uma temperatura de $120^{\circ} \mathrm{C}$ e concentrações de maltodextrina de $15 \%$, obteve pó não higroscópico (<10\%) e de muita formação de caking $(78,36 \%)$.

Segundo Goula e Adamopoulos (2010), o caking de um pó alimentício depende de sua higroscopicidade, uma vez que a formação de caking em pós alimentícios ricos em açúcares pode ser atribuída à absorção de umidade. A água é adsorvida na superfície das partículas, formando uma solução saturada e tornando as partículas pegajosas. 
Os resultados de higroscopicidade e grau de caking para a polpa de graviola em pó nas condições estudadas evidenciaram a necessidade da investigação mais profunda sobre seu comportamento higroscópico durante seu armazenamento.

Os parâmetros dos modelos ajustados para as isotermas da polpa de graviola em pó, além dos valores dos coeficientes de determinação $\left(R^{2}\right)$ e erros médios relativos $\mathrm{E}(\%)$, estão presentes na Tabela 4 .

Os modelos matemáticos utilizados apresentaram altos coeficientes de determinação e baixos erros médios relativos para a maioria dos ajustes. Geralmente, considera-se um modelo com ajuste aceitável quando apresenta erros médios relativos menores que 10\% (PEDRO et al., 2010; MOREIRA et al., 2013; OLIVEIRA et al., 2013a, 2014; ROCHA et al., 2014) e Labuza et al. (1985) relatam que a representação de isotermas é extremamente boa quando o erro relativo for menor que $5 \%$, uma vez que estes são os parâmetros utilizados para definir o modelo que melhor se ajusta a este estudo.

Neste trabalho, o modelo de GAB forneceu o melhor ajuste para a polpa de graviola em pó obtida por secagem

Tabela 4. Parâmetros dos modelos matemáticos para representação das isotermas de sorção da polpa de graviola em pó formulada com $17 \%(\mathrm{~m} / \mathrm{m})$ de maltodextrina e obtida por secagem em spray dryer em $169^{\circ} \mathrm{C}$ de temperatura.

\begin{tabular}{|c|c|c|c|c|c|c|}
\hline \multirow{2}{*}{ Modelo } & \multirow{2}{*}{$\mathrm{T}\left({ }^{\circ} \mathrm{C}\right)$} & \multicolumn{3}{|c|}{ Parâmetros } & \multirow{2}{*}{$\mathbf{R}^{2}$} & \multirow{2}{*}{$E(\%)$} \\
\hline & & $\mathbf{X}_{\mathrm{m}}$ & C & K & & \\
\hline \multirow{4}{*}{ 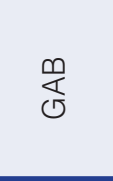 } & 25 & 0,666 & 0,189 & 0,724 & 0,999 & 2,97 \\
\hline & 30 & 0,747 & 0,170 & 0,723 & 0,998 & 0,58 \\
\hline & 35 & 0,669 & 0,193 & 0,730 & 0,999 & 1,84 \\
\hline & 40 & 0,750 & 0,176 & 0,730 & 0,999 & 4,47 \\
\hline & & $X_{m}$ & C & N & & \\
\hline \multirow{4}{*}{$\bar{\varpi}$} & 25 & 1,532 & 0,036 & 4,927 & 0,999 & 5,22 \\
\hline & 30 & 1,439 & 0,039 & 4,987 & 0,999 & 5,96 \\
\hline & 35 & 1,165 & 0,049 & 5,075 & 0,999 & 5,25 \\
\hline & 40 & 1,240 & 0,048 & 5,050 & 0,999 & 4,27 \\
\hline \multirow{5}{*}{ 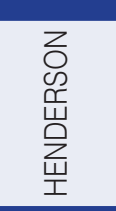 } & & a & b & & & \\
\hline & 25 & 0,751 & 3,799 & & 0,998 & 4,27 \\
\hline & 30 & 0,739 & 3,673 & & 0,998 & 6,18 \\
\hline & 35 & 0,738 & 3,600 & & 0,999 & 4,18 \\
\hline & 40 & 0,728 & 3,451 & & 0,998 & 1,35 \\
\hline & & $\mathbf{a}$ & b & & & \\
\hline \multirow{4}{*}{ 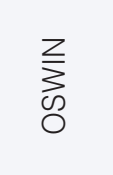 } & 25 & 0,111 & 0,719 & & 0,991 & 13,24 \\
\hline & 30 & 0,112 & 0,738 & & 0,990 & 9,03 \\
\hline & 35 & 0,114 & 0,746 & & 0,992 & 12,06 \\
\hline & 40 & 0,116 & 0,767 & & 0,992 & 3,74 \\
\hline
\end{tabular}

$\mathrm{R}^{2}$ : coeficiente de determinação; $E(\%)$ : erro médio relativo; $X$ : conteúdo de água na monocamada molecular ( $\mathrm{g}$ de $\mathrm{H}_{2} \mathrm{O} \mathrm{g}^{-1}$ de matéria seca); C: constante relativa ao calor total de sorçấo da primeira camada; $\mathrm{K}$ : constante de GAB relativa às multicamadas; $\mathrm{N}$ : Constante de BET relativa às multicamadas; a e b: parâmetros de ajuste para os modelos de Henderson e Os. em spray dryer, com coeficientes de determinação $\left(R^{2}\right)$ maiores que 0,99 e erros médios (E) menores que 5\%.

Segundo Pavan et al. (2012), os modelos de GAB e BET baseiam-se no conceito de umidade na monocamada, um parâmetro importante para avaliação da estabilidade dos alimentos. O conteúdo de umidade na monocamada $\left(X_{m}\right)$ expressa em $\mathrm{g}$ de $\mathrm{H}_{2} \mathrm{O} \cdot \mathrm{g}^{-1}$ de matéria seca e corresponde à quantidade de água na monocamada, ou seja, fortemente adsorvida a sítios específicos na superfície dos alimentos, não participa de reações, é de difícil eliminação pela secagem e também não é congelável por estar fortemente ligada ao produto (ALVES et al., 2015). É considerado um valor crítico, acima do qual as taxas de algumas reações de degradação aumentam e a estabilidade da matriz alimentar diminui (COMUNIAN et al., 2011; OLIVEIRA et al., 2013a).

Observou-se que houve um aumento da umidade na monocamada $\left(X_{m}\right)$ quando a temperatura subiu de 25 a $40^{\circ} \mathrm{C}$. Comportamento similar é relatado por Ferreira e Pena (2003), Moreira et al. (2013) e Moura Neto et al. (2015), cujos modelos GAB e BET tiveram um aumento em $\mathrm{X}_{\mathrm{m}}$ entre temperaturas de $25^{\circ} \mathrm{C}$ a $40^{\circ} \mathrm{C}$ em estudos sobre o comportamento higroscópico de farinha de palmeira de pêssego, manga liofilizada e polpa de cajá em pó obtida por secagem em spray dryer, respectivamente. Ferreira e Pena (2003) relatam ainda que, apesar deste comportamento não ser muito comum, dois mecanismos podem ser utilizados para justificá-lo. Um aumento na temperatura pode provocar modificações na estrutura física do produto, disponibilizando um maior número de sítios ativos com afinidade entre moléculas de água ou um aumento na solubilidade de solutos intrínsecos ao produto, resultando em um maior número de moléculas de água retido na monocamada. Sendo a solubilidade uma consequência do fenômeno de solvatação.

Na temperatura de $25^{\circ} \mathrm{C}$, o valor de $X_{m}$ de $0,666 \mathrm{~g}$ de $\mathrm{H}_{2} \mathrm{O} \mathrm{g}^{-1}$ de matéria seca, quando convertido para base úmida (OLIVEIRA et al., 2013b), representa uma umidade de $66 \%$, indicando o teor de umidade para armazenamento do material visando menores alterações deteriorantes.

Em outras palavras, observou-se que a polpa de graviola em pó obtida por secagem em spray dryer requer cuidados quando armazenada em ambiente com umidade relativa superior a $66 \%$ e, quando expostas a ambientes assim, recomenda-se que seu armazenamento seja realizado com o auxílio de embalagens que ofereçam resistências a troca de umidade.

Com relação ao parâmetro $C$ do modelo de $G A B$, que representa o calor total de sorção da primeira camada e tem significado físico relacionado ao efeito da temperatura (MORAGA et al., 2006; ROCHA et al., 2014), observou-se tendência à diminuição do valor com o aumento da temperatura de $25^{\circ} \mathrm{C}$ para $40^{\circ} \mathrm{C}$ (Tabela 4). Semelhante aos comportamentos observados por Moraga et al. (2006), 
Rocha et al. (2014) e Moura Neto et al. (2015) em seus estudos sobre o comportamento higroscópico da polpa de kiwi liofilizada e das polpas de manga e cajá em pó adicionadas de maltodextrina e obtidas por secagem em spray dryer, respectivamente.

O parâmetro C do modelo GAB auxiliou ainda a predizer o formato e o tipo de isoterma do material, pois segundo Gogus et al. (1998), valores inferiores a 10 para a Constante $\mathrm{C}$ indicam curvas de isoterma em formato de $\mathrm{J}$ classificadas como tipo III.

$\mathrm{O}$ valor da Constante $\mathrm{K}$ de GAB representa uma medida das interações entre as moléculas do adsorvato com o adsorvente (CATELAM et al, 2011). Desta maneira, de acordo com Goula et al. (2008) seus valores devem ser menores que 1,0 .

Observou-se, de acordo com a Tabela 4, que a força de interação entre o vapor de água e a matriz sólida da polpa de graviola em pó aumentou, a partir da elevação da temperatura, partindo de 0,72 na temperatura de $25^{\circ} \mathrm{C}$ e chegando a valores de 0,73 na temperatura de $40^{\circ} \mathrm{C}$.

As isotermas de adsorção de umidade para a polpa de graviola em pó nas temperaturas de $25^{\circ} \mathrm{C}, 30^{\circ} \mathrm{C}$, $35^{\circ} \mathrm{C}$ e $40^{\circ} \mathrm{C}$ foram construídas pelo ajuste ao modelo $\mathrm{GAB}$ (Figura 1). Conforme o esperado, o comportamento das isotermas foi do tipo exponencial, apresentando formato do tipo III (formato de J), característica de alimentos ricos em açúcar (HÉBRARD et al., 2003).

De acordo com Blahovec (2004), para o modelo de GAB e para as curvas do tipo III, os valores aceitáveis para as Constantes são $0<\mathrm{K} \leq 1$ e $0 \leq \mathrm{C} \leq 1$. Na Tabela 4 observou-se que estes parâmetros estão dentro do intervalo descrito, tendo assim, a confirmação matemática do tipo de isotermas obtido nesse estudo.

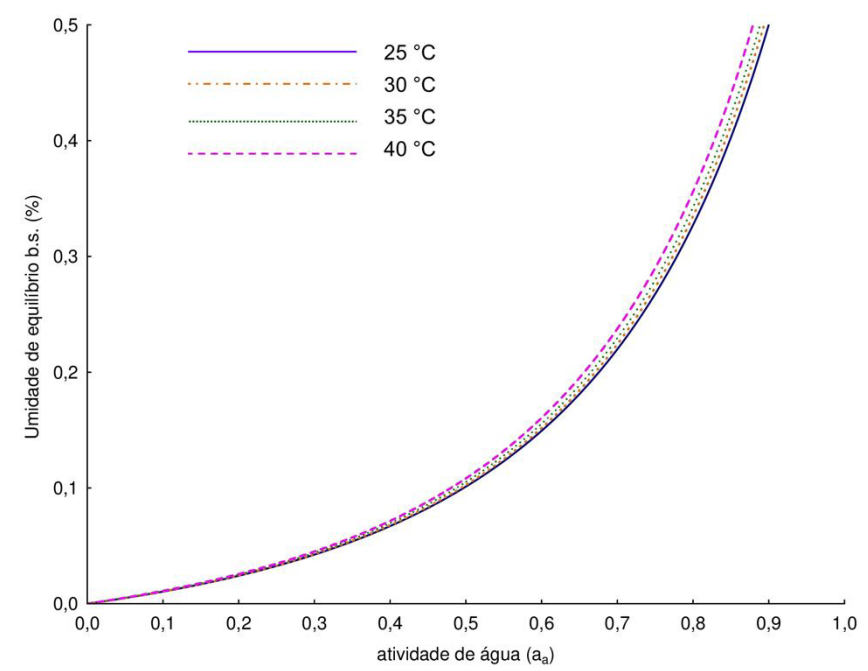

Figura 1. Isotermas de adsorção de acordo com o modelo GAB a $25^{\circ} \mathrm{C}, 30^{\circ} \mathrm{C}, 35^{\circ} \mathrm{C}$ e $40^{\circ} \mathrm{C}$ para a polpa de graviola em pó obtida por secagem em spray dryer, em $169^{\circ} \mathrm{C}$ de temperatura e $17 \%(\mathrm{~m} / \mathrm{m})$ de maltodextrina como adjuvante de secagem.
Por intermédio da Figura 1, pode-se notar que as isotermas apresentam condições de atividade de água e umidade de equilíbrio muito próximas devido à estreita faixa de temperatura avaliada $\left(25^{\circ} \mathrm{C}\right.$ para $\left.40^{\circ} \mathrm{C}\right)$ e que a partir dos valores de atividade de água de 0,3, um aumento da temperatura resulta em um aumento na umidade de equilíbrio para uma mesma atividade de água. Pedro et al. (2010) relatam que este comportamento pode ser explicado pelo aumento da solubilidade dos açúcares em água, devido à temperatura.

O mesmo tipo de isoterma e o mesmo comportamento frente ao aumento da temperatura são relatados por outros autores em estudos com diversas polpas de frutas em pó formuladas com maltodextrina e obtidas por secagem em spray dryer (SANTOS et al., 2014; ROCHA et al., 2014; MOURA NETO et al., 2015), levando a crer que este comportamento seja típico para polpas de frutas em pó obtidas pelo mesmo processo.

Tal comportamento fica ainda mais evidente a partir de valores de atividade de água de 0,6, no qual um pequeno aumento na atividade de água resulta em um grande ganho de umidade pelo pó. Este resultado indica que é necessário ter cuidado no armazenamento da polpa de graviola em pó em ambientes com umidade relativa acima de 60\%. Desta maneira, reforçando as conclusões obtidas com relação ao valor de umidade da monocamada $\left(X_{m}\right)$ de $\mathrm{GAB}$ a $25^{\circ} \mathrm{C}$ (Tabela 4) que indicaram $66 \%$ como sendo o teor limite de umidade relativa do ambiente de armazenamento para este material.

\section{Conclusões}

A polpa de graviola em pó formulada com 17\% (m/m) de maltodextrina e obtida por secagem em spray dryer a $169^{\circ} \mathrm{C}$ pode ser considerada ligeiramente higroscópica e de muita formação de caking. Suas isotermas foram mais bem representadas pelo modelo GAB, apresentaram comportamento do tipo III e revelaram que um aumento da temperatura em uma mesma atividade de água resulta em aumentos no teor de umidade do pó. Por fim, recomenda-se o armazenamento da polpa de graviola em pó em ambientes com umidade relativa menor que $60 \%$ e ressalta-se que acima desse valor a estabilidade do material passa a ser comprometida.

\section{Referências}

ALEXANDRE, H. V.; FIGUEIREDO, R. M. F.; QUEIROZ, A. J. M. Moisture adsorption isotherms of red brazilian cherry powder. Revista de Biologia e Ciências da Terra, v. 7, p. 11-20, 2007.

ALVES, T. P.; FÓZ, H. D.; NICOLETI, J. F. Isotermas de dessorção de pimentão verde e energia envolvida no processo. Brazilian Journal of Food Technology, v. 18, n. 2, p. 137-145, 2015. http://dx.doi.org/10.1590/1981-6723.6114. 
Comportamento higroscópico da polpa de graviola em pó obtida por secagem em spray dryer

Cavalcante, C. E. B. et al.

BLAHOVEC, J. Sorption isotherms in materials of biological origin mathematical and physical approach. Journal of Food Engineering, v. 65, n. 4, p. 489-495, 2004. http://dx.doi. org/10.1016/j.jfoodeng.2004.02.012

BRASIL. Ministério da Agricultura, Pecuária e Abastecimento. Instrução normativa $n^{\circ} 1$, de 7 de janeiro de 2000. Dispõe sobre o regulamento técnico geral para fixação dos padrões de identidade e qualidade para polpa de fruta, Diário Oficial [da] República Federativa do Brasil, Brasília, DF, 7 jan. 2000.

CANO-CHAUCA, M.; STRINGHETA, P. C.; RAMOS, A. M.; CAL-VIDAL, $\mathrm{J}$. Effect of the carriers on the microstructure of mango powder obtained by spray drying and its functional characterization. Innovative Food Science \& Emerging Technologies, v. 6, n. 4, p. 420-428, 2005. http://dx.doi.org/10.1016/j.ifset.2005.05.003.

CAPARINO, O. A.; TANG, J.; NINDO, C. I.; SABLANI, S. S.; POWERS, J. R.; FELLMAN, J. K. Effect of drying methods on the physical properties and microstructures of mango (Philippine 'Carabao' var.) powder. Journal of Food Engineering, v. 111, n. 1, p. 135-148, 2012. http://dx.doi.org/10.1016/j.jfoodeng.2012.01.010.

CATELAM, K. T.; TRINDADE, C. S. F.; ROMERO, J. T. Water adsorption isotherms and isosteric sorption heat of spray-dried and freeze-dried dehydrated passion fruit pulp with additives and skimmed milk. Ciência e Agrotecnologia, v. 35, n. 6, p. 11961203, 2011. http://dx.doi.org/10.1590/S1413-70542011000600021.

CAVAlCANTE, C. E. B.; RODRIGUES, S.; AFOnSO, M. R. A.; COSTA, J. M. C. Avaliação dos parâmetros de secagem da polpa de graviola em pó obtida por secagem em spray dryer. Brazilian Journal of Food Technology, v. 20, e2016015, 2017.

CebAllos, A. M.; GIRALDO, G. I.; ORREGO, C. E. Effect of freezing rate on quality parameters of freeze dried soursop fruit pulp. Journal of Food Engineering, v. 111, n. 2, p. 360-365, 2012. http://dx. doi.org/10.1016/j.jfoodeng.2012.02.010.

CHEGINI, G. R.; GHOBADIAN, B. Effect of spray-drying conditions on physicalproperties of orange juice powder. Drying Technology, v. 23, n. 3, p. 657-668, 2005. http://dx.doi. org/10.1081/DRT-200054161.

COMUNIAN, T. A.; MONTERREY-QUINTERO, E. S.; THOMAZINI, M.; BALIEIRO, J. C. C.; PICCONE, P.; PITTIA, P.; FAVARO-TRINDADE, C. S. Assessment of production efficiency, physicochemical properties and storage stability of spray-dried chlorophyllide, a natural food colourant, using gum Arabic, maltodextrin and soy protein isolate-based carrier systems. International Journal of Food Science \& Technology, v. 46, n. 6, p. 1259-1265, 2011. http://dx.doi.org/10.1111/j.1365-2621.2011.02617.x.

COSTA, J. P.; ROCHA, É. M. F. F.; COSTA, J. M. C. Study of the physicochemical characteristics of soursop powder obtained by spray-drying. Food Science and Technology, v. 34, n. 4, p. 663-666, 2014. http://dx.doi.org/10.1590/1678-457X.6380.
CUNNIFF, P. (Ed.). Official methods of analysis of the Association of Official Analytical Chemists. 16th ed. Gaithersburg: AOAC, 1997. v. 2, p. 16-17.

FAZAELI, M.; EMAM-DJOMEH, Z.; KALBASI ASHTARI, A.; OMID, $M$. Effect of spray drying conditions and feed composition on the physical properties of black mulberry juice powder. Food and Bioproducts Processing, v. 90, n. 4, p. 667-675, 2012. http:// dx.doi.org/10.1016/j.fbp.2012.04.006.

FERRARI, C. C.; RIBEIRO, C. P.; AGUIRRE, J. M. Secagem por atomização de polpa de amora-preta usando maltodextrina como agente carreador. Brazilian Journal of Food Technology, v. 15, n. 2, p. 157-165, 2012. http://dx.doi.org/10.1590/S198167232012005000009

FERREIRA, C. D.; PENA, R. S. Comportamento higroscópico da farinha de pupunha (Bactris gasipaes). Food Science and Technology, v. 23, n. 2, p. 251-255, 2003. http://dx.doi. org/10.1590/S0101-20612003000200025.

GEA NIRO RESEARCH LABORATORY. GEA Niro analytical methods: analytical methods dry milk products. 14th ed. Søborg: GEA, 2003. 103 p

GOGUS, F.; MASKAN, M.; KAYA, A. Sorption isotherms of turkish delight. Journal of Food Protection, v. 22, p. 345-357, 1998.

GOULA, A. M.; ADAMOPOULOS, K. G. A new technique for spray drying orange juice concentrate. Innovative Food Science \& Emerging Technologies, v. 11, n. 2, p. 342-351, 2010. http:// dx.doi.org/10.1016/j.ifset.2009.12.001.

GOULA, A. M.; KARAPANTSIOS, T. D.; ACHILIAS, D. S.; ADAMOPOULOS, K. G. Water sorption isotherms and glass transition temperature of spray dried tomato pulp. Journal of Food Engineering, v. 85, n. 1, p. 73-83, 2008. http://dx.doi. org/10.1016/j.jfoodeng.2007.07.015

GREENSPAN, L. Humidity fixed points of binary satured aqueous solutions. Journal of Research of the National Bureau of Standards Physics and Chemistry, v. 81A, n. 1, p. 89-96, 1977. http://dx.doi.org/10.6028/jres.081A.011

HÉBRARD, A.; OULAHNA, D.; GALET, L.; CUQ, B.; ABECASSIS, J.; FAGES, J. Hydration properties of durum wheat semolina: influence of particle size. Powder Technology, v. 130, n. 1-3, p. 211-218, 2003. http://dx.doi.org/10.1016/S0032-5910(02)00268-1.

INSTITUTO ADOLFO LUTZ - IAL. Analytical standards of the Institute Adolfo Lutz: chemical and physical methods for food analysis. 4. ed. São Paulo: IAL, 2008. 1020 p.

JAYA, S.; DAS, H. Effect of maltodextrin, glycerol monostearate and tricalcium phosphate on vaccum dried mango powders properties. Journal of Food Engineering, v. 63, n. 2, p. 125-134, 2004. http://dx.doi.org/10.1016/S0260-8774(03)00135-3.

LABUZA, T. P.; KAANANE, A.; CHEN, J. Y. Effects of temperature on moisture sorption isotherms and water activity shift of two dehydrated foods. Journal of Food Science, v. 50, n. 2, p. 
Comportamento higroscópico da polpa de graviola em pó obtida por secagem em spray dryer

Cavalcante, C. E. B. et al.

385-392, 1985. http://dx.doi.org/10.1111/j.1365-2621.1985. tb13409.x.

LEMOS, E. E. P. The production of annona fruits in Brazil. Revista Brasileira de Fruticultura, v. 36, n. 1, p. 77-85, 2014. http:// dx.doi.org/10.1590/S0100-29452014000500009.

LIMA, M. A. C.; ALVES, R. E.; FILGUEIRAS, H. A. C. Comportamento respiratório e amaciamento da graviola (Annona muricata L.) após tratamentos pós-colheita com cera e 1-metilciclopropeno. Ciência e Agrotecnologia, v. 34, n. 1, p. 155-162, 2010. http:// dx.doi.org/10.1590/S1413-70542010000100020.

MORAGA, G.; MARTINEZ-NAVARRETE, N.; CHIRALT, A. Water sorption isotherms and phase transitions in kiwifruit. Journal of Food Engineering, v. 72, n. 2, p. 147-156, 2006. http://dx.doi. org/10.1016/j.jfoodeng.2004.11.031

MOREIRA, T. B.; ROCHA, E. M. F. F.; AFONSO, M. R. A.; COSTA, J. M. C. Comportamento das isotermas de adsorção do pó da polpa de manga liofilizada. Revista Brasileira de Engenharia Agrícola e Ambiental, v. 17, n. 10, p. 1093-1098, 2013. http:// dx.doi.org/10.1590/S1415-43662013001000011.

MOURA NETO, L. G.; ROCHA, É. M. F. F.; AFONSO, M. R. A.; COSTA, J. M. C. Adsorption isotherms of hog plum (Spondias mombin L.) pulp powder obtained by spray dryer. Acta Scientiarum, v. 37, n. 2, p. 273-277, 2015. http://dx.doi.org/10.4025/actascitechnol. v37i2.25001.

OLIVEIRA, D. M.; CLEMENTE, E.; AFONSO, M. R. A.; COSTA, J. M. C. Hygroscopic behavior of lyophilized powder of grugru palm (Acrocomia aculeata). American Journal of Analytical Chemistry, v. 4, n. 10, p. 1-7, 2013a. http://dx.doi.org/10.4236/ ajac.2013.410A3001.

OLIVEIRA, D. M.; CLEMENTE, E.; COSTA, J. M. C. Hygroscopic behavior and degree of caking of grugru palm (Acrocomia aculeata) powder. Journal of Food Science and Technology, v. 51, n. 10, p. 2783-2789, 2014. http://dx.doi.org/10.1007/ s13197-012-0814-9. PMid:25328226.

OLIVEIRA, M. I. S.; TONON, R. V.; NOGUEIRA, R. I.; CABRAL, L. M. C. Estabilidade da polpa de morango atomizada utilizando diferentes agentes carreadores. Brazilian Journal of Food Technology, v. 16, n. 4, p. 310-318, 2013b. http://dx.doi. org/10.1590/S1981-67232013005000037.

PAVAN, M. A.; SCHMIDT, S. J.; FENG, H. Water sorption behavior and thermal analysis of freeze-dried, refractance window dried and hot-air dried açaí (Euterpe oleracea Martius) juice. LebensmittelWissenschaft + Technologie, v. 48, n. 1, p. 75-81, 2012. http:// dx.doi.org/10.1016/j.Iwt.2012.02.024.

PEDRO, M. A. M.; TELIS-ROMERO, J.; TELIS, V. R. N. Effect of drying method on the adsorption isotherms and isosteric heat of passion fruit pulp powder. Food Science and Technology, v. 30, n. 4, p. 993-1000, 2010. http://dx.doi.org/10.1590/S010120612010000400024.

QUEK, S. Y.; CHOK, N. K.; SWEDLUND, P. The physicochemical properties of spray-dried watermelon powder. Chemical Engineering and Processing, v. 46, n. 5, p. 386-392, 2007. http://dx.doi.org/10.1016/j.cep.2006.06.020.

ROCHA, E. M. F. F.; RODRIGUES, S.; AFONSO, M. R. A.; COSTA, J. M. C. Mathematical modeling for isotherms of mango pulp powder, obtained by atomization. Journal of Encapsulation and Adsortion Science, v. 4, n. 01, p. 8-14, 2014. http://dx. doi. org/10.4236/jeas.2014.41002.

SANTOS, A. A. C.; FLORÊNCIO, A. K. G. D.; ROCHA, E. M. F. F.; COSTA, J. M. C. Physicochemical evaluation and hygroscopic behavior of powdered guava obtained by spray drying. Revista Ciência Agronômica, v. 45, n. 3, p. 508-514, 2014. http://dx. doi. org/10.1590/S1806-66902014000300010.

STATSOFT. Statsoft statistica for windows: computer programa manual. Version 7.0. Tulsa: Statsoft Inc., 2007.

TONON, R. V.; BRABET, C.; HUBINGER, M. D. Influência da temperatura do ar de secagem e da concentração de agente carreador sobre as propriedades físico-químicas do suco de açaí em pó. Revista Ciência e Tecnologia de Alimentos, v. 29, n. 2, p. 444-450, 2009. http://dx.doi.org/10.1590/S010120612009000200034. 